\title{
ABOUT THE APPROXIMATE SOLUTION OF THE USUAL AND GENERALIZED HILBERT BOUNDARY VALUE PROBLEMS FOR ANALYTICAL FUNCTIONS
}

V.R. KRISTALINSKII

Smolensk State Pedagogical University

214000 Smolensk, Russia

Received November 6, 1998; revised June 10, 1999

\begin{abstract}
In this article the methods for obtaining the approximate solution of usual and generalized Hilbert boundary value problems are proposed. The method of solution of usual Hilbert boundary value problem is based on the theorem about the representation of the kernel of the corresponding integral equation by $\tau=t$ and on the earlier proposed method for the computation of the Cauchy-type integrals. The method for approximate solution of the generalized boundary value problem is based on the method for computation of singular integral of the form$$
\frac{1}{2 \pi i} \int_{L} \frac{A(t, \tau)}{\tau-\tau_{1}} d \tau
$$

proposed by the author. All methods are implemented with the Mathcad and Maple.

\section{FORMULATION OF THE PROBLEM}

Let $D$ be the region, bounded by a simple closed curve $L$. It is known, that the Hilbert boundary value problem can be formulated so: obtain analytical in $D^{+}$and continuous on the boundary function

$$
f(z)=u(x, y)+i v(x, y)
$$

real and imaginary parts of which satisfy on the boundary the equation

$$
a(s) u(s)+b(s) v(s)=c(s),
$$


where $a(s), b(s)$ and $c(s)$ are real functions of the arc length of the contour and on the contour they satisfy the Hollder condition. There are some methods for the solution of this problem. One of them is given in [1]. The general solution of the problem (1.1) is given by the formula

$$
\varphi(z)=z_{1}^{m} \mathrm{X}_{10}(z)\left(\frac{1}{2 \pi i} \int_{L} \frac{\mu_{0}(\tau)}{\tau-z}+\sum_{j=0}^{\kappa_{1}} \beta_{10}(z) w_{j 0}(z)\right),
$$

where $\mu_{0}(t)$ is the solution of the Fredholm's integral equation

$$
\begin{aligned}
Z \mu_{0}(t) & \equiv \mu_{0}(t)+\frac{1}{2 \pi i} \int_{L}\left(\frac{1}{\tau-t}-\frac{\overline{\tau^{\prime 2}(s)}}{\bar{\tau}-\bar{t}}\right) \mu_{0}(\tau) d \tau \\
= & \frac{Q_{1}(t)}{t^{m} \mathrm{X}_{10}(t)}, \\
\mathrm{X}_{10}^{+}(z) & =\exp \left\{\frac{1}{2 \pi i} \int_{L} \frac{\gamma_{10}(\tau}{\tau-z} d \tau\right\},
\end{aligned}
$$

$\gamma_{10}$ is the solution of the integral equation

$$
\begin{aligned}
& Z \gamma_{10}(t)=\ln \left[\frac{t^{-} m}{t^{m}} G(t)\right] \\
& Q(t)=\frac{2 c(t)}{a(t)-i b(t)}, \quad G(t)=-\frac{a(t)+i b(t)}{a(t)-i b(t)},
\end{aligned}
$$

and $\frac{m}{2}$ is the index of the problem. The functions $w(z)$ are expressed by the solutions of the Fredholm integral equations. One of the approximate methods for the solution of this problem is proposed in [2]. But the numerical experiment shows, that this method is unstable and its error grows near the boundary. This article proposes the method for the approximate solution of the Hilbert boundary value problem. It is based on the method of approximate computation of the Cauchy-type integral, proposed by the author in [3].

\section{FORMULATION OF THE ALGORITHM}

Firstly we will prove the following theorem.

Theorem 2.1. Let a simple smooth curve $L$ be parametrized $x=x(f), y=$ $y(f)$, where $x(f), y(f)$ are three times differentiable, $2 \pi$ - periodical functions. We define the function

$$
K\left(f_{1}, f_{2}\right)=\frac{1}{\tau-t}-\frac{\overline{t^{2}(s)}}{\bar{\tau}-\bar{t}}
$$


where $\tau\left(f_{1}\right)=x\left(f_{1}\right)+i y\left(f_{1}\right), t\left(f_{2}\right)=x\left(f_{2}\right)+i y\left(f_{2},\right) s$ is the natural parameter. $K$ is a continuous function, if at $\tau=t$ it is defined as

$$
K\left(f_{1}, f_{1}\right)=\frac{1}{2 \overline{t^{\prime}(s)}} \frac{y^{\prime}\left(f_{1}\right) x^{\prime \prime}\left(f_{1}\right)-x^{\prime}\left(f_{1}\right) y^{\prime \prime}\left(f_{1}\right)}{\left(\left(x^{\prime}\left(f_{1}\right)\right)^{2}+\left(y^{\prime}\left(f_{1}\right)^{2}\right)^{\frac{3}{2}}\right.} .
$$

Proof. Because $s$ is the natural parameter, then $\left|\tau^{\prime}(s)\right|=1$. Hence

$$
\frac{1}{\tau-t}-\frac{\overline{\tau^{\prime 2}(s)}}{\bar{\tau}-\bar{t}}=\frac{\overline{\tau^{\prime}(s)} \tau^{\prime}(s)}{\tau-t}-\frac{\overline{\tau^{\prime 2}(s)}}{\tau-t}=\overline{\tau^{\prime}(s)} 2 i \operatorname{Im} \frac{\tau^{\prime}(s)}{\tau-t}
$$

It can be simply proved, that

$$
\begin{aligned}
\operatorname{Im} \frac{\tau^{\prime}(s)}{\tau-t}= & \frac{1}{\sqrt{\left(x^{\prime}\left(f_{1}\right)\right)^{2}+\left(\left(y^{\prime}\left(f_{1}\right)\right)^{2}\right.}} \\
& \frac{y^{\prime}\left(f_{1}\right)\left(x\left(f_{1}\right)-x\left(f_{2}\right)\right)-x^{\prime}\left(f_{1}\right)\left(y\left(f_{1}\right)-y\left(f_{2}\right)\right)}{\left(x\left(f_{1}\right)-x\left(f_{2}\right)\right)^{2}\left(y\left(f_{1}\right)-y\left(f_{2}\right)\right)^{2}} .
\end{aligned}
$$

Let $f_{2}=f_{1}+\Delta f$. Using the Taylor formula with the Peano residual term we can obtain

$$
\begin{aligned}
& x\left(f_{2}\right)=x\left(f_{1}\right)+x^{\prime}\left(f_{1}\right) \Delta f+\frac{x^{\prime \prime}\left(f_{1}\right)}{2 !}+o(\Delta f)^{2}, \\
& y\left(f_{2}\right)=y\left(f_{1}\right)+y^{\prime}\left(f_{1}\right) \Delta f+\frac{y^{\prime \prime}\left(f_{1}\right)}{2 !}+o(\Delta f)^{2} .
\end{aligned}
$$

Substituting $x\left(f_{2}\right), y\left(f_{2}\right)$ in (1.4) and passing to the limit at $\Delta f \rightarrow 0$ we obtain

$$
\lim _{\Delta f \rightarrow 0} \frac{I m \tau^{\prime}(s)}{\tau-t}=\frac{1}{2} \frac{y^{\prime}\left(f_{1}\right) x^{\prime \prime}\left(f_{1}\right)-x^{\prime}\left(f_{1}\right) y^{\prime \prime}\left(f_{1}\right)}{\left(\left(x^{\prime}\left(f_{1}\right)\right)^{2}+\left(y^{\prime}\left(f_{1}\right)^{2}\right)^{\frac{3}{2}}\right.} .
$$

We propose the following algorithm for the solution of the Hilbert boundary value problem:

1. Obtain the index of the problem $m$.

2. Compute the function $\ln \left[\frac{t^{-m}}{t^{m}} G(t(f))\right]$.

3. Solve the integral equation (1.5). We obtain the discretization of the kernel, using the theorem, formulated above.

4. In order to define the function $\gamma_{10}(z)$, we construct spline interpolation of the solution of the integral equation (1.5), obtain $k$-th partial sum of the Fourier series of the function and using this sum, construct the function $g_{1}(t)$, where $t \in L$. 
5. Compute the Cauchy-type integral

$$
\frac{1}{2 \pi i} \int_{L} \frac{g_{1}(\tau)}{\tau-z} .
$$

We find the function $w(z)$, which maps the region $D^{+}$on the unity circle conformally, and the reverse function, mapping the unity circle on $D^{+}$conformally. Next we expand the function $g_{1}\left(z\left(e^{i \varphi}\right)\right)$ in Fourier series and consider $k$-th partial sum of this series. We consider the function $\varphi_{1}(w(z))$ and obtain $L_{k}(z)$ - the main part of the Laurent series of this function. The approximate value of the Cauchy-type integral of this function is the function $\varphi(z)-L_{k}(z)$. This function is continuous in the closed region up to the contour $L$. Let define the function $\mathrm{X}^{+}(z)$. Then we compute the approximate solution of the integral equation (1.2), construct its spline interpolation, approximate it by the $k$-th partial sum of its Fourier series and compute the Cauchy-type integral as described above. Constants in (1.5) can be defined by

$$
\begin{gathered}
W_{0}(z) \equiv 1, W_{2 k-1}(z)=\frac{1}{z^{k}}+\frac{1}{2 \pi i} \int_{L} \frac{\nu_{2 k-1}(\tau)}{\tau-z} d \tau \\
W_{2 k}(z)=\frac{1}{z^{k}}+\frac{1}{2 \pi i} \int_{L} \frac{\nu_{2 k}(\tau)}{\tau-z} d \tau
\end{gathered}
$$

where $\nu_{i}(t)$ are solutions of Fredholm's integral equations $\left(z \nu_{i}\right)(t)=q_{i}(t)$ and

$$
q_{2 k-1}(t)=\frac{1}{\bar{t}^{k}}-\frac{1}{t^{k}}, q_{2 k}(t)=-\frac{i}{\bar{t}^{k}}-\frac{i}{t^{k}} .
$$

This algorithm was realized in MathCad. In the numerical experiment we take $x(f)=a \cos (f)+i b \sin (f)$ and consider the boundary value problem. We obtain the analytic in $D^{+}$function, whose boundary values satisfy the equation

$$
\begin{gathered}
\cos (0.2 \cos (f)) u(f)+\sin (0.2 \cos (f)) v(f)=\operatorname{Re} \sin (a \cos (f)+i b \sin (f)) \\
\cos (0.2 \cos (f))+I m \sin (a \cos (f)+i b \sin (f)) \sin (0.2 \cos (f)) .
\end{gathered}
$$

The approximate value of the function, mapping the ellipse onto the unity circle is given in [4]:

$$
\begin{gathered}
z(w)=\left(1-\frac{1}{8} \lambda^{2}+\frac{3}{128} \lambda^{4}\right) w\left(1+\left(\frac{1}{2} \lambda-\frac{1}{4} \lambda^{3}+\frac{3}{32} \lambda^{5}\right) w^{2}\right. \\
\left.+\left(\frac{1}{2} \lambda^{2}-\frac{9}{16} \lambda^{4}\right) w^{4}+\left(\frac{5}{9} \lambda^{3}-\frac{9}{8} \lambda^{5}\right) w^{6}+\frac{7}{8} \lambda^{4} w^{8}+\frac{21}{16} \lambda^{5} w^{10}\right),
\end{gathered}
$$


where $\lambda=0.2$. For the approximation of the inverse function we use the method, proposed by L.V. Kantorowich in [ 5] and developed by G.A. Nikolaeva in [6]. This method can be implemented with Maple. The index of this problem is 0 , and one of its solutions is $\sin (z)$. For example, at $z=0.7$ the value of $\sin (z)=0.644217687$ and the value of the obtained approximate solution is $0.64141183-0.00063675 i$.

\section{THE GENERALIZED HILBERT PROBLEM}

The generalized Hilbert boundary value problem can be formulated as follows. Let $D$ be a finite simply connected region, bounded by Liapunov's contour $L$. It is required to obtain the analytic single valued function $\varphi(x, i)=u(x, y)+$ $i v(x, y)$, continuous in $D^{+} \cup L$, which satisfies on $L$ the equation

$$
\operatorname{Re}\left\{[a(t)-i b(t)] \varphi^{+}(t)+\int_{L} A_{1}(t, \tau) \varphi^{+}(\tau) d \tau+\int_{L} B_{1}(t, \tau) \overline{\varphi^{+}(\tau)} d \tau\right\}=c(t),
$$

where $a(t), b(t), c(t)$ are given on $L$ real functions from $H(L)$ and kernels $A_{1}(t, \tau), B_{1}(t, \tau)$ are given functions from $H(L \times L)$. We shall suppose, that the contour can be parametrized as $t=t(\varphi)$, where $t(\varphi)$ is sufficiently times differentiable function. It is known, that the solution of this problem has the following form:

$$
\varphi(z)=\frac{1}{2 \pi i} \int_{L} \frac{\mu(z)}{\overline{\lambda(t)}} \frac{d \tau}{\tau-z}+\sum_{k=1}^{m} c_{k} z^{k},
$$

where $\mu(t)$ is a solution of Fredholm integral equation

$$
\mu(t)+\int_{L} N(t, \tau) \mu(\tau) d \sigma=2 c(t)-\sum_{k=1}^{2 m+1} a_{k} \omega_{k}(t)
$$

$m$ is the index of the problem, functions $\omega_{k}(t)$ have a form

$$
\begin{gathered}
\omega_{2 k-1}=2 \operatorname{Im}\left\{\overline{\lambda(t)} t^{k-1}+\int_{L} A_{1}(t, \tau) \tau^{k-1} d \tau+\int_{L} B_{1}(t, \tau) \bar{t} a u^{k-1} d \tau\right\}, \\
\omega_{2 k}=2 \operatorname{Re}\left\{\overline{\lambda(t)} t^{k-1}+\int_{L} A_{1}(t, \tau) \tau^{k-1} d \tau-\int_{L} B_{1}(t, \tau) \bar{t} a u^{k-1} d \tau\right\} .
\end{gathered}
$$

In this case the kernel of the equation has a form

$$
\operatorname{Re}\left\{\frac{1}{2 \pi i}\left[\frac{\lambda(\tau)}{\lambda(t)} \frac{\tau^{\prime}(\sigma)}{\tau-t}\right]+A_{1}(t, \tau) \lambda(\tau) \tau^{\prime}(\sigma)+\frac{B_{1}(t, \tau) \tau^{\prime}(\sigma)}{\lambda(t)}\right.
$$




$$
+\frac{\tau^{\prime}(\sigma) \lambda(\tau)}{\pi i} \int_{L} \frac{A_{1}\left(t, \tau_{1}\right)}{\tau-\tau_{1}} d \tau_{1}-\overline{\lambda(\tau)} \frac{\overline{\tau^{\prime}(\sigma)}}{\pi i} \int_{L} \frac{B_{1}\left(t, \tau_{1}\right)}{\bar{\tau}-\bar{\tau}_{1}} d \bar{\tau}_{1} .
$$

The following singular integrals enter this equation:

$$
\frac{1}{\pi i} \int_{L} \frac{A_{1}\left(t, \tau_{1}\right)}{\tau-\tau_{1}} d \tau, \quad \frac{1}{\pi i} \int_{L} \frac{B_{1}\left(t, \tau_{1}\right)}{\tau-\tau_{1}} d \tau .
$$

For the approximation of this integrals we propose the method of generalized trigonometric series. Let the contour $L$ be parametrized as $t=t(\varphi)$, where $0 \leq$ $\varphi \leq 2 \pi$. First we expand functions $A_{1}\left(t(\varphi), \tau\left(\varphi_{1}\right)\right), B_{1}\left(t(\varphi), \tau\left(\varphi_{1}\right)\right)$ in Fourier series and replace obtained series by their partial sums. For the function $A_{1}\left(t(\varphi), \tau\left(\varphi_{1}\right)\right)$ the partial sum of its Fourier series has a form

$$
S_{m}\left(\varphi, \varphi_{1}\right)=\frac{a_{0}(\varphi)}{2}+\sum_{k=1}^{m} a_{k}(\varphi) \cos \left(k \varphi_{1}\right)+b_{k} \sin \left(k \varphi_{1}\right),
$$

where coefficients of the series are given by

$$
a_{k}=\frac{1}{2 \pi} \int_{0}^{2 \pi} \tilde{A}\left(\varphi, \varphi_{1}\right) \cos \left(k \varphi_{1}\right), \quad b_{k}=\frac{1}{2 \pi} \int_{0}^{2 \pi} \tilde{A}\left(\varphi, \varphi_{1}\right) \sin \left(k \varphi_{1}\right) .
$$

For the function $B_{1}\left(t(\varphi), \tau\left(\varphi_{1}\right)\right)$ the partial sum of its Fourier series has a form

$$
S 1_{m}\left(\varphi, \varphi_{1}\right)=\frac{a 1_{0}(\varphi)}{2}+\sum_{k=1}^{m} a 1_{k}(\varphi) \cos \left(k \varphi_{1}\right)+b 1_{k} \sin \left(k \varphi_{1}\right),
$$

where coefficients of the series are given by

$$
a 1_{k}=\frac{1}{2 \pi} \int_{0}^{2 \pi} \tilde{A}\left(\varphi, \varphi_{1}\right) \cos \left(k \varphi_{1}\right), \quad b 1_{k}=\frac{1}{2 \pi} \int_{0}^{2 \pi} \tilde{A}\left(\varphi, \varphi_{1}\right) \sin \left(k \varphi_{1}\right) .
$$

We approximate the singular integral

$$
\frac{1}{2 \pi i} \int_{L} \frac{A_{1}(t, \tau)}{\tau-\tau_{1}} d \tau
$$

by the equation

$$
\frac{1}{\pi i} \int_{L} \frac{A_{1}\left(t(\varphi), \tau\left(\varphi_{1}\right)\right)}{\tau-\tau_{1}} d \varphi_{1} \approx \frac{1}{\pi i} \int_{L} \frac{S_{m}\left(t(\varphi), \tau\left(\varphi_{1}\right)\right)}{\tau-\tau_{1}} d \varphi_{1}
$$




$$
\begin{aligned}
& =\frac{a_{0}(\varphi)}{2} \frac{1}{\pi i} \int_{L} \frac{d \tau}{\tau-\tau_{1}}+\sum_{k=1}^{m} a_{k}(\varphi) \frac{1}{\pi i} \int_{L} \frac{\cos \left(k \varphi_{1}\right)}{\tau-\tau_{1}} d \tau \\
& +\sum_{k=1}^{m} b_{k}(\varphi) \frac{1}{\pi i} \int_{L} \frac{\sin \left(k \varphi_{1}\right)}{\tau-\tau_{1}} d \tau .
\end{aligned}
$$

We can describe each of the singular integrals

$$
\frac{1}{\pi i} \int_{L} \frac{\cos \left(k \varphi_{1}\right)}{\tau-\tau_{1}} d \tau, \quad \frac{1}{\pi i} \int_{L} \frac{\sin \left(k \varphi_{1}\right)}{\tau-\tau_{1}} d \tau
$$

using Sochotzki-Plemelj formula:

$$
\begin{aligned}
& \frac{1}{\pi i} \int_{L} \frac{\cos \left(k \varphi_{1}\right)}{\tau-\tau_{1}} d \tau=2 \Phi^{+}\left(\cos \left(k \varphi_{1}\right)\right)-\cos \left(k \varphi_{1}\right), \\
& \frac{1}{\pi i} \int_{L} \frac{\sin \left(k \varphi_{1}\right)}{\tau-\tau_{1}} d \tau=2 \Phi^{+}\left(\sin \left(k \varphi_{1}\right)\right)-\sin \left(k \varphi_{1}\right),
\end{aligned}
$$

where $\Phi^{+}\left(\cos \left(k \varphi_{1}\right)\right)$ and $\Phi^{+}\left(\sin \left(k \varphi_{1}\right)\right)$ are boundary values of corresponding Cauchy-type integrals. Analogously we can approximate the integral

$$
\frac{1}{2 \pi i} \int_{L} \frac{B_{1}(t, \tau)}{\tau-\tau_{1}} d \tau .
$$

We can compute the first component of the kernel, using the theorem, formulated above. Then we approximately solve the integral equation, reducing it to the linear system and obtain the solution of the problem, computing the Cauchy-type integral, as described above.

\section{REFERENCES}

[1] F.D. Gachov. Boundary value problems. Nauka, Moscow, 1977. (in Russian)

[2] M.A. Aleksidze. Fundamental solutions for solving boundary value problems. Nauka, Moscow, 1991. (in Russian)

[3] V.R. Kristalinskii. An approximate method for computation of Cauchy-type integrals. In: Proceedings The 20-th Summer School "Applications of Mathematics to Engineering", The 20-th Summer School "Applications of Mathematics to Engineering",Varna, 1994.

[4] V.I. Smirnov. A course on mathematics. Moscow, 1957. (in Russian)

[5] L.V. Kantorovich, V.I. Krylov. Approximate methods of the analysis. Fizmatgiz, Leningrad, 1962. (in Russian)

[6] G.A. Nikolajeva. Trudy Mat. Instituta AN SSSR. 53, 236-265. 


\section{APIE KLASIKINIO IR APIBENDRINTO HILBERTO KRASTTINIŲ UZ̃DAVINIŲ SKAITINI SPRENDIMA}

\section{V.R. KRISTALINSKII}

Pateikti du skaitiniai metodai klasikinio ir apibendrinto Hilberto kraštinių uždavinių sprendimui. Pirmasis metodas skirtas klasikinio uždavinio sprendimui, jis remiasi teorema apie atitinkamos integralinès lygties branduolio skleidimą taško $\tau=t$ aplinkoje ir Košy tipo integralų skaičiavimo metodais. Apibendrintojo uždavinio sprendimo metodas remiasi metodu, kuris buvo skirtas skaičiuoti singuliarius integralus

$$
\frac{1}{2 \pi i} \int_{L} \frac{A(t, \tau)}{\tau-\tau_{1}} d t .
$$

Metodai realizuoti Maple ir Mathcad paketais. 